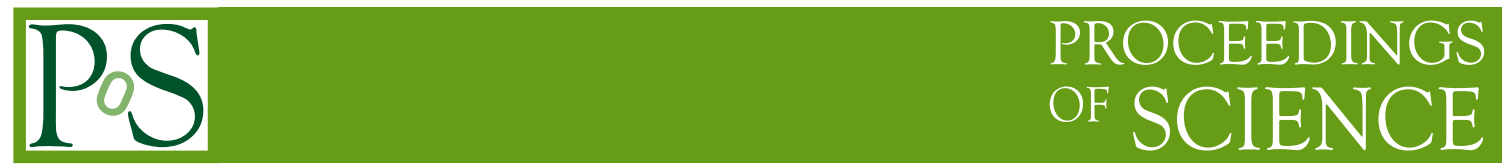

\title{
Direct Reactions for Nuclear Astrophysics
}

\author{
C.A. Bertulani* ${ }^{\dagger}$ \\ Department of Physics and Astronomy, Texas AE M University-Commerce, Commerce TX 75429, \\ USA \\ E-mail: carlos.bertulani@tamuc.edu
}

The reactions of relevance for stellar evolution are difficult to measure directly in the laboratory at the small astrophysical energies. In recent years indirect reaction methods have been developed and applied to extract low-energy astrophysical S-factors. These methods require a combination of new experimental techniques and theoretical efforts, which are the subject of this review.

51 st International Winter Meeting on Nuclear Physics,

21-25 January 2013

Bormio, Italy

\footnotetext{
*Speaker.

${ }^{\dagger}$ The author acknowledges financial support from the US Department of Energy Grants DE-FG02- 08ER41533, DE-SC0004971.
} 


\section{Introduction}

\subsection{Fusion}

Fusion cross sections can be calculated from the equation [1]

$$
\sigma_{F}(E)=\pi \hbar^{2} \sum_{\ell}(2 \ell+1) P_{\ell}(E)
$$

where $E$ is the center of mass energy, $\lambda=\sqrt{\hbar^{2} / 2 m E}$ is the reduced wavelength and $\ell=0,1,2, \cdots$. The cross section is proportional to $\pi t^{2}$, the area of the quantum wave. Each part of the wave corresponds to different impact parameters having different probabilities for fusion. As the impact parameter increases, so does the angular momentum, hence the reason for the $2 \ell+1$ term. $P_{\ell}(E)$ is the probability that fusion occurs at a given impact parameter, or angular momentum. Sometimes, for a better visualization, or for extrapolation to low energies, one uses the concept of astrophysical $S$-factor, redefining the cross section as

$$
\sigma_{F}(E)=\frac{1}{E} S(E) \exp [-2 \pi \eta(E)],
$$

where $\eta(E)=Z_{1} Z_{2} e^{2} / \hbar v$, with $v$ being the relative velocity. The exponential function is an approximation to $P_{0}(E)$ for a square-well nuclear potential plus Coulomb potential, whereas the factor $1 / E$ is proportional to the area appearing in Eq. 1.1.

\subsection{Many reaction channels}

Eq. 1.1 does not work in most situations. Only by including coupling to other channels, the fusion cross sections can be reproduced. In coupled channels schemes one expands the total wavefunction for the system as

$$
\Psi=\sum_{i} a_{i}(\alpha) \phi_{i}\left(\alpha, q_{k}\right)
$$

where $\phi$ form the channel basis, $\alpha$ is a dynamical variable (e.g., the distance between the nuclei), and $q_{k}$ are intrinsic coordinates. Inserting this expansion in the Schrödinger equation yields a set of CC equations in the form [2]

$$
\frac{d a_{k}}{d \alpha}=\sum_{j} a_{j}\left\langle\phi_{k}|U| \phi_{j}\right\rangle e^{i E_{\alpha} \alpha / \hbar}
$$

where $U$ is whatever potential that couples the channels $k$ and $j$ and $E_{\alpha}=E_{\alpha}^{(k)}-E_{\alpha}^{(j)}$ is some sort of transition energy, or transition momentum. In the presence of continuum states, continuumcontinuum coupling (relevant for breakup channels) can be included by discretizing the continuum. This goes by the name of Continuum Discretized Coupled-Channels (CDCC) calculations. There are several variations of CC equations, e.g., a set of differential equations for the wavefunctions, instead of using basis amplitudes. Coupled channels calculations with a large number of channels in continuum couplings are somewhat challenging: the phases of matrix elements can add destructively or constructively, depending on the system and on the nuclear model. Such suppressions or enhancements are difficult to interpret. 


\subsection{Radiative capture}

For reactions involving light nuclei, only a few channels are of relevance. In this case, a real potential is enough for the treatment of fusion. For example, radiative capture cross sections of the type $n+x \rightarrow a+\gamma$ and $\pi L(\pi=E,(M)=$ electric (magnetic) L-pole) transitions can be calculated from [3]

$$
\sigma_{E L, J_{b}}^{\text {d.c. }} \propto\left|\left\langle l_{c} j_{c}\left\|O_{\pi L}\right\| l_{b} j_{b}\right\rangle\right|^{2},
$$

where $O_{\pi L}$ is an EM operator, and $\left\langle l_{c} j_{c}\left\|O_{\pi L}\right\| l_{b} j_{b}\right\rangle$ is a multipole matrix element involving bound (b) and continuum (c) wavefunctons. For electric multipole transitions $\left(O_{\pi L}=r^{L} Y_{L M}\right)$,

$$
\left\langle l_{c} j_{c}\left\|O_{E L}\right\| l_{b} j_{b}\right\rangle \propto \int_{0}^{\infty} d r r^{L} u_{b}(r) u_{c}(r)
$$

where $u_{i}$ are radial wavefunctions. The total direct capture cross section is obtained by adding all multipolarities and final spins of the bound state $\left(E \equiv E_{n x}\right)$,

$$
\sigma^{\text {d.c. }}(E)=\sum_{L, J_{b}}(S F)_{J_{b}} \sigma_{E L, J_{b}}^{\text {d.c. }}(E),
$$

where $(S F)_{J_{b}}$ are spectroscopic factors.

\subsection{Asymptotic normalization coefficients}

In a microscopic approach, instead of single-particle wavefunctions one often makes use of overlap integrals, $I_{b}(r)$, and a many-body wavefunction for the relative motion, $u_{c}(r)$. Both $I_{b}(r)$ and $u_{c}(r)$ might be very complicated to calculate, depending on how elaborated the microscopic model is. The variable $r$ is the relative coordinate between the nucleon and the nucleus $x$, with all the intrinsic coordinates of the nucleons in $x$ being integrated out. The direct capture cross sections are obtained from the calculation of $\sigma_{L, J_{b}}^{\text {d.c. }} \propto\left|\left\langle I_{b}(r)\left\|r^{L} Y_{L}\right\| \Psi_{c}(r)\right\rangle\right|^{2}$.

The imprints of many-body effects will eventually disappear at large distances between the nucleon and the nucleus. One thus expects that the overlap function asymptotically matches $(r \rightarrow$ $\infty)$

$$
I_{b}(r)=C_{i} \times\left(\frac{W_{-\eta, l_{b}+1 / 2}(2 \kappa r)}{r}\right)\left[\sqrt{\frac{2 \kappa}{r}} K_{l_{b}+1 / 2}(\kappa r)\right],
$$

where () are for protons and [] for neutrons. The binding energy of the $n+x$ system is related to $\kappa$ by means of $E_{b}=\hbar^{2} \kappa^{2} / 2 m_{n x}, W_{p, q}$ is the Whittaker function and $K_{\mu}$ is the modified Bessel function. In Eq. 1.8, $C_{i}$ is the Asymptotic Normalization Coefficient (ANC).

In the calculation of $\sigma_{L, J_{b}}^{\text {d.c. }}$ above, one often meets the situation in which only the asymptotic part of $I_{b}(r)$ and $\Psi_{c}(r)$ contributes significantly to the integral over $r$. In these situations, $u_{c}(r)$ is also well described by a simple two-body scattering wave (e.g. Coulomb waves). Therefore the radial integration in $\sigma_{L, J_{b}}^{\text {d.c. }}$ can be done accurately and the only remaining information from the many-body physics at short-distances is contained in the asymptotic normalization coefficient $C_{i}$, i.e. $\sigma_{L, J_{b}}^{\text {d.c. }} \propto C_{i}^{2}$. We thus run into an effective theory for radiative capture cross sections, in which the constants $C_{i}$ carry all the information about the short-distance physics, where the many-body aspects are relevant [4] . It is worthwhile to mention that these arguments are reasonable for proton capture at very low energies, because of the Coulomb barrier. 


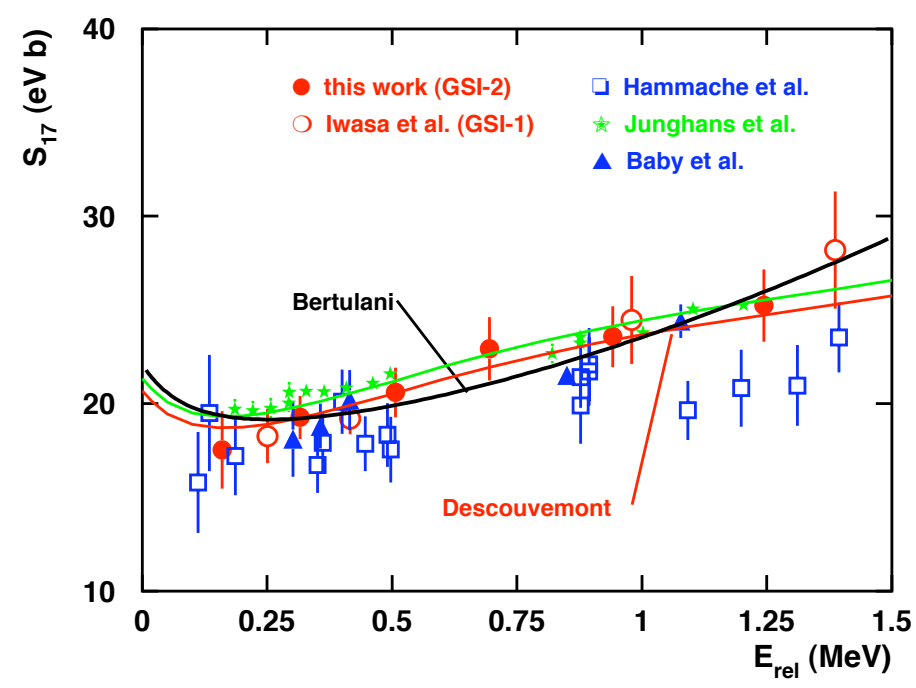

Figure 1: World data on ${ }^{7} \mathrm{Be}(\mathrm{p}, \gamma)^{8} \mathrm{~B}$ compared to theoretical calculations [7]. The solid curve is from Eq. (1.10).

\subsection{Resonating group method}

One immediate goal can be achieved in the coming years by using the Resonating Group Method (RGM) or the Generator Coordinate Method (GCM). These are a set of coupled integrodifferential equations of the form [5]

$$
\sum_{\alpha^{\prime}} \int d^{3} r^{\prime}\left[H_{\alpha \alpha^{\prime}}^{A B}\left(\mathbf{r}, \mathbf{r}^{\prime}\right)-E N_{\alpha \alpha^{\prime}}^{A B}\left(\mathbf{r}, \mathbf{r}^{\prime}\right)\right] g_{\alpha^{\prime}}\left(\mathbf{r}^{\prime}\right)=0
$$

where $H(N)\left(\mathbf{r}, \mathbf{r}^{\prime}\right)=\left\langle\Psi_{A}(\alpha, \mathbf{r})|H(1)| \Psi_{B}\left(\alpha^{\prime}, \mathbf{r}^{\prime}\right)\right\rangle$. In these equations $H$ is the Hamiltonian for the system of two nuclei (A and B) with the energy $E, \Psi_{A, B}$ is the wavefunction of nucleus A (and B), and $g_{\alpha}(\mathbf{r})$ is a function to be found by numerical solution of Eq. 1.9, which describes the relative motion of A and B in channel $\alpha$. Full antisymmetrization between nucleons of A and B are implicit. Modern nuclear shell-model calculations are able to provide the wavefunctions $\Psi_{A, B}$ for light nuclei [6].

The astrophysical S-factor for the reaction ${ }^{7} \mathrm{Be}(\mathrm{p}, \gamma){ }^{8} \mathrm{~B}$ was calculated [8] and excellent agreement was found with the experimental data in both direct and indirect measurements $[8,9]$. The low- and high-energy slopes of the S-factor obtained with a many-body microscopic calculation [8] is well described by the fit [7]

$$
S_{17}(E)=(22.109 \mathrm{eV} . \mathrm{b}) \frac{1+5.30 E+1.65 E^{2}+0.857 E^{3}}{1+E / 0.1375}
$$

where $\mathrm{E}$ is the relative energy (in $\mathrm{MeV}$ ) of $\mathrm{p}+{ }^{7} \mathrm{Be}$ in their center-of-mass. This equation corresponds to a Padé approximant of the $\mathrm{S}$-factor. A subthreshold pole due to the binding energy of ${ }^{8} \mathrm{~B}$ is responsible for the denominator $[10,11]$. Figure 1 shows the world data on ${ }^{7} \mathrm{Be}(\mathrm{p}, \gamma)^{8} \mathrm{~B}$ compared 
to a few of the theoretical calculations. The recent compilation published in Ref. [12] recommends $S_{17}=20.8 \pm 0.7$ (expt) \pm 1.4 (theor) eV b.

\section{Direct reactions and the role of radioactive beams}

\subsection{Transfer reactions}

Transfer reactions are effective when a momentum matching exists between the transferred particle and the internal particles in the nucleus. Thus, beam energies should be in the range of a few $10 \mathrm{MeV}$ per nucleon. Low energy reactions of astrophysical interest can be extracted directly from breakup reactions $a+b \longrightarrow s+c+C$ by means of the Trojan Horse method (THM) [13]. If the Fermi momentum of the particle $x$ inside $b=(s+x)$ compensates for the initial projectile velocity $v_{b}$, the low energy reaction $a+x \longrightarrow c+C$ is induced at very low (even vanishing) relative energy between $a$ and $x$. Basically, this technique extends the method of transfer reactions to continuum states. Very successful results using this technique have been reported [14, 15].

\subsubsection{The Trojan Horse Method}

The THM was successfully applied to study several two-body reactions relevant for astrophysical applications by using appropriate three-body breakup reactions. The method has proven to be particularly suited for acquiring information on charged- as well as neutral-particle-induced reaction cross sections at astrophysical energies, since it allows the particles to overcome, in the case of charged-particle-induced reactions, the Coulomb barrier of the two-body entrance channel. THM allows one to extract the low-energy behavior of a binary reaction by applying the well-known theoretical formalism of the quasi-free $(\mathrm{QF})$ process. The basic idea of the THM is to extract the cross section in the low-energy region of a two-body reaction with significant astrophysical impact,

$$
a+x \longrightarrow c+C
$$

from a suitable three-body QF reaction,

$$
a+b \longrightarrow s+c+C
$$

An example of application of the THM is the $\mathrm{S}$-factor for the ${ }^{2} \mathrm{H}(\mathrm{d}, \mathrm{p})^{3} \mathrm{H}$ reaction extracted through ${ }^{3} \mathrm{He}$ breakup in the ${ }^{2} \mathrm{H}\left({ }^{3} \mathrm{He}, \mathrm{pt}\right) \mathrm{H}$ reaction.

The assumption is that of an interaction between the impinging nucleus and one of the clusters constituting the target (called participant $x$, a deuteron in the present case), while the residual nucleus does not participate in the reaction (spectator $s,{ }^{4} \mathrm{He}$ or $\mathrm{p}$ in the two different cases). The latter is free from any effect due to the interaction between the incoming nucleus and the participants, reflecting in the exit channel the same momentum distribution, for the inter-cluster $(x-s)$ motion inside $b$, it had before the occurrence of the QF breakup. QF processes are direct mechanisms in which the interaction between an impinging nucleus and the target can cause the target breakup or the projectile breakup. In particular, these processes have three particles in the exit channel, one of which can be thought as a spectator to the binary interaction of interest. Under appropriate kinematical conditions, the three-body reaction $a(b, c C) s$ is considered as the decay of the Trojan horse $b$ into the clusters $x$ and s followed by the interaction of $a$ with $x$. If the bombarding energy 
$E_{a}$ is chosen high enough to overcome the Coulomb barrier in the entrance channel of the reaction, the effect of the Coulomb barrier and electron screening effects are negligible. The application of the THM significantly simplifies if the PWIA is valid. In this approach the triple differential cross section in the center of mass of the $\mathrm{TH}$ reaction can be written as

$$
\frac{d^{3} \sigma}{d E_{c} d \Omega_{c} d \Omega_{C}} \propto K_{F}\left|\Phi\left(p_{s x}\right)\right|^{2} \sum_{l_{l}}\left|L_{l_{l}}\right|^{2}\left(\frac{d \sigma_{l_{l}}}{d \Omega_{c . m .}}\right)^{H O E S},
$$

where

(i) $l_{l}$ is the orbital angular momentum of particles $s$ and $x$ in the entry channel of the binary subreaction and $L_{l}$ is a function of relative momentum and kinetic energy in the entry channel of the binary sub-reaction as defined in Ref. [16];

(ii) $\left.(d \sigma / d \Omega)_{c m}\right)^{H O E S}$ is the half-off-energy-shell (HOES) differential cross section for the twobody reaction at the center-of-mass energy $E_{c m}$ given in post-collision prescription by

$$
E_{c m}=E_{c-C}-Q_{2 b}
$$

where $Q_{2 b}$ is the two-body $Q$-value of the binary process and $E_{c-C}$ is the relative energy between the outgoing particles;

(iii) $K_{F}$ is a kinematical factor containing the final-state phase-space factor and is a function of the masses, momenta, and angles of the outgoing particles;

(iv) $\Phi\left(p_{s x}\right)$ is the Fourier transform of the radial wave function $\chi(r)$ for the $x-s$ intercluster motion, usually described in terms of Hänkel, Eckart, and Hulthën functions depending on the $x-s$ system properties.

The success of the THM relies on the QF kinematics (equivalent to $\Phi\left(p_{s x}\right) \sim 0$ for nuclei like ${ }^{3} \mathrm{He}$ or ${ }^{2} \mathrm{H}$ where the dominant wave of the inter-cluster relative motion is $l=0$ ), at which the $\mathrm{TH}$ conditions are best fulfilled. The occurrence of the QF mechanism at low energies has been pointed out in a number of papers $[17,18,19,20]$. The TH triple differential cross section can be written in a factorized form, as in Eq. (2.3) in terms of the HOES differential cross section whose energy trend is the relevant information for the THM. Its absolute value can be extracted through normalization by the direct data available at higher energies. Thus, if the PWIA is valid, the HOES differential cross section for the binary sub-reaction determined from the TH reaction should not depend on the type of the TH nucleus, as was proven in Refs. [21] for the two examined cases (see also, Fig. 2).

\subsubsection{The ANC method}

Another transfer method, coined as ANC technique [4,23,24] relies on fact that the amplitude for the radiative capture cross section $b+x \longrightarrow a+\gamma$ is given by

$$
M=<I_{b x}^{a}\left(\mathbf{r}_{\mathbf{b x}}\right)\left|O\left(\mathbf{r}_{\mathbf{b x}}\right)\right| \psi_{i}^{(+)}\left(\mathbf{r}_{\mathbf{b x}}\right)>
$$

where

$$
I_{b x}^{a}=<\phi_{a}\left(\xi_{b}, \xi_{x}, \mathbf{r}_{\mathbf{b x}}\right) \mid \phi_{x}\left(\xi_{x}\right) \phi_{b}\left(\xi_{b}\right)>
$$

is the integration over the internal coordinates $\xi_{b}$, and $\xi_{x}$, of $b$ and $x$, respectively. For low energies, the overlap integral $I_{b x}^{a}$ is dominated by contributions from large $r_{b x}$. Thus, what matters for the 


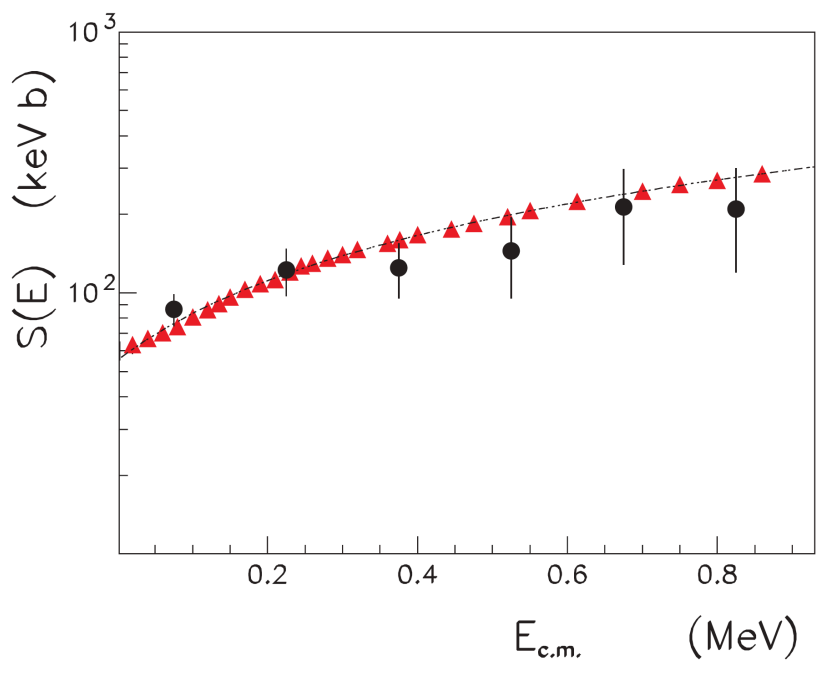

Figure 2: Averaged astrophysical $\mathrm{S}(\mathrm{E})$ factor for the $\mathrm{d}(\mathrm{d}, \mathrm{p}) \mathrm{t}$ reaction measured via THM after ${ }^{6} \mathrm{Li}$ breakup (black dots) and after $3 \mathrm{He}$ breakup(red points), extracted from Ref. [22], clearly showing the Trojan horse particle invariance. The polynomial fit to data from Ref. [22] is reported for comparison as a solid line.

calculation of the matrix element $M$ is the asymptotic value of $I_{b x}^{a} \sim C_{b x}^{a} W_{-\eta_{a}, 1 / 2}\left(2 \kappa_{b x} r_{b x}\right) / r_{b x}$, where $C_{b x}^{a}$ is the ANC and $W$ is the Whittaker function. This coefficient is the product of the spectroscopic factor and a normalization constant which depends on the details of the wave function in the interior part of the potential. Thus, $C_{b x}^{a}$ is the only unknown factor needed to calculate the direct capture cross section. These normalization coefficients can be found from: 1) analysis of classical nuclear reactions such as elastic scattering [by extrapolation of the experimental scattering phase shifts to the bound state pole in the energy plane], or 2) peripheral transfer reactions whose amplitudes contain the same overlap function as the amplitude of the corresponding astrophysical radiative capture cross section. One of the many advantages of using transfer reaction techniques over direct measurements is to avoid the treatment of the electron screening problem [14, 24].

\subsection{Intermediate energy Coulomb excitation}

The Coulomb excitation cross section is given by

$$
\frac{d \sigma_{i \rightarrow f}}{d \Omega}=\left(\frac{d \sigma}{d \Omega}\right)_{\mathrm{el}} \frac{16 \pi^{2} Z_{2}^{2} e^{2}}{\hbar^{2}} \sum_{\pi \lambda \mu} \frac{B\left(\pi \lambda, I_{i} \rightarrow I_{f}\right)}{(2 \lambda+1)^{3}}|S(\pi \lambda, \mu)|^{2},
$$

where $B\left(\pi \lambda, I_{i} \rightarrow I_{f}\right)$ is the reduced transition probability of the projectile nucleus, $\pi \lambda=E 1, E 2$, $M 1, \ldots$ is the multipolarity of the excitation, and $\mu=-\lambda,-\lambda+1, \ldots, \lambda$.

The relativistic corrections to the Rutherford formula for $(d \sigma / d \Omega)_{\mathrm{el}}$ (relevant for collisions at $50 \mathrm{MeV} /$ nucleon and above) has been investigated in Ref. [25]. It was shown that the scattering angle increases by up to $6 \%$ when relativistic corrections are included in nuclear collisions at 100 $\mathrm{MeV} /$ nucleon. The effect on the elastic scattering cross section is even more drastic: up to $13 \%$ for center-of-mass scattering angles around 0-4 degrees.

The orbital integrals $S(\pi \lambda, \mu)$ contain the information about relativistic corrections. Inclusion of absorption effects in $S(\pi \lambda, \mu)$ due to the imaginary part of an optical nucleus-nucleus potential where worked out in Ref. [26]. These orbital integrals depend on the Lorentz factor $\gamma=\left(1-v^{2} / c^{2}\right)^{-1 / 2}$, with $c$ being the speed of light, on the multipolarity $\pi \lambda \mu$, and on the adiabatic- 
ity parameter $\xi(b)=\omega_{f i} b / \gamma v<1$, where $\omega_{f i}=\left(E_{f}-E_{i}\right) / \hbar$ is the excitation energy (in units of $\hbar$ ) and $b$ is the impact parameter.

Ref. [27] has shown that at $10 \mathrm{MeV} /$ nucleon the relativistic corrections are important only at the level of $1 \%$. At $500 \mathrm{MeV} /$ nucleon, the correct treatment of the recoil corrections is relevant on the level of $1 \%$. Thus the non-relativistic treatment of Coulomb excitation [28] can be safely used for energies below about $10 \mathrm{MeV} /$ nucleon and the relativistic treatment with a straight-line trajectory [29] is adequate above about $500 \mathrm{MeV} /$ nucleon. However at energies around 50 to 100 $\mathrm{MeV} /$ nucleon, accelerator energies common to most radioactive beam facilities, it is very important to use a correct treatment of recoil and relativistic effects, both kinematically and dynamically. At these energies, the corrections can add up to 50\%. These effects were also shown in Ref. [30] for the case of excitation of giant resonances in collisions at intermediate energies.

A reliable extraction of useful nuclear properties, like the electromagnetic response (B(E2)values, $\gamma$-ray angular distribution, etc.) from Coulomb excitation experiments at intermediate energies requires a proper treatment of special relativity [27, 31]. The dynamical relativistic effects have often been neglected in the analysis of experiments elsewhere (see, e.g. [32]). The effect is highly non-linear, i.e. a $10 \%$ increase in the velocity might lead to a 50\% increase (or decrease) of certain physical observables. A general review of the importance of the relativistic dynamical effects in intermediate energy collisions has been presented in Ref. [2, 33].

\subsection{The Coulomb dissociation method}

The Coulomb dissociation method is quite simple. The (differential, or angle integrated) Coulomb breakup cross section for $a+A \longrightarrow b+c+A$ follows from Eq. 2.5. It can be rewritten as

$$
\frac{d \sigma_{C}^{\pi \lambda}(\omega)}{d \Omega}=N^{\pi \lambda}(\omega ; \theta ; \phi) . \sigma_{\gamma+a \rightarrow b+c}^{\pi \lambda}(\omega)
$$

where $\omega$ is the energy transferred from the relative motion to the breakup, and $\sigma_{\gamma+a \rightarrow b+c}^{\pi \lambda}(\omega)$ is the photo nuclear cross section for the multipolarity $\pi \lambda$ and photon energy $\omega$. The function $N^{\pi \lambda}$, sometimes called virtual photon numbers, depends on $\omega$, the relative motion energy, nuclear charges and radii, and the scattering angle $\Omega=(\theta, \phi) . N^{\pi \lambda}$ can be reliably calculated [34] for each multipolarity $\pi \lambda$. Time reversal allows one to deduce the radiative capture cross section $b+c \longrightarrow a+\gamma$ from $\sigma_{\gamma+a \rightarrow b+c}^{\pi \lambda}(\omega)$. This method was proposed in Ref. [35] and has been tested successfully in a number of reactions of interest for astrophysics. The most celebrated case is the reaction ${ }^{7} \mathrm{Be}(\mathrm{p}, \gamma)^{8} \mathrm{~B}$ [36], followed by numerous experiments in the last decade (see e.g. Ref. [37]).

Eq. 2.6 is based on first-order perturbation theory. It also assumes that the nuclear contribution to the breakup is small, or that it can be separated under certain experimental conditions. The contribution of the nuclear breakup has been examined by several authors (see, e.g. [38]). ${ }^{8} \mathrm{~B}$ has a small proton separation energy $(\approx 140 \mathrm{keV})$. For such loosely-bound systems it had been shown that multiple-step, or higher-order effects, are important [39]. These effects occur by means of continuum-continuum transitions. Detailed studies of dynamic contributions to the breakup were explored in refs. [40, 41] and in several other publications which followed. The role of higher multipolarities (e.g., E2 contributions $[3,42,43]$ in the reaction $\left.{ }^{7} \mathrm{Be}(\mathrm{p}, \gamma)^{8} \mathrm{~B}\right)$ and the coupling to high-lying states has also to be investigated carefully. It has also been shown that the influence of giant resonance states is small [44]. 


\subsection{Knock-out reactions}

The early interest in knockout reactions came from studies of nuclear halo states, for which the narrow momentum distributions of the core fragments in a qualitative way revealed the large spatial extension of the halo wave function. It was shown [45] that the longitudinal component of the momentum (taken along the beam or $z$ direction) gave the most accurate information on the intrinsic properties of the halo and that it was insensitive to details of the collision and the size of the target. In contrast to this, the transverse distributions of the core are significantly broadened by diffractive effects and by Coulomb scattering. For experiments that observe the nucleon produced in elastic breakup, the transverse momentum is entirely dominated by diffractive effects, as illustrated [46] by the angular distribution of the neutrons from the reaction ${ }^{9} \mathrm{Be}\left({ }^{11} \mathrm{Be},{ }^{10} \mathrm{Be}+\mathrm{n}\right) \mathrm{X}$. In this case, the width of the transverse momentum distribution reflects essentially the size of the target [47].

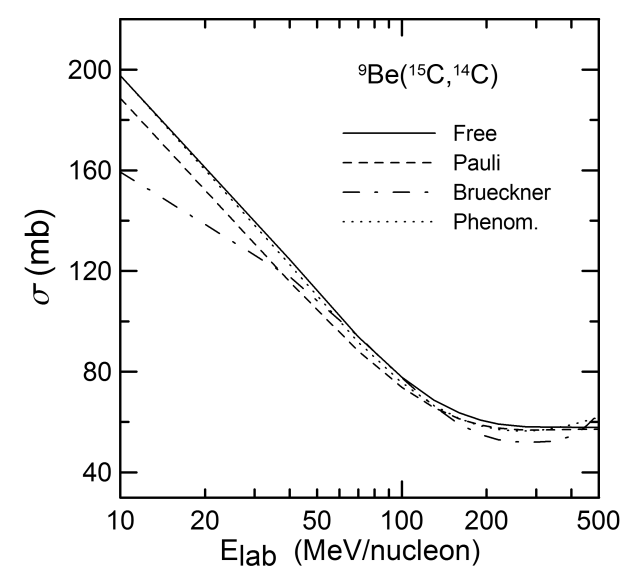

Figure 3: Total knockout cross sections for removing the $l=0$ halo neutron of ${ }^{15} \mathrm{C}$, bound by $1.218 \mathrm{MeV}$, in the reaction ${ }^{9} \mathrm{Be}\left({ }^{15} \mathrm{C},{ }^{14} \mathrm{C}_{g s}\right)$. The solid curve is obtained with the use of free nucleon-nucleon cross sections. The dashed curve includes the geometrical effects of Pauli blocking. The dashed-dotted curve is the result using the Brueckner theory, and the dotted curve is a phenomenological parametrization [48].

To test the influence of the medium effects in nucleon knockout reactions, we consider the removal of the $l=0$ halo neutron of ${ }^{15} \mathrm{C}$, bound by $1.218 \mathrm{MeV}$. The reaction studied is ${ }^{9} \mathrm{Be}\left({ }^{15} \mathrm{C},{ }^{14} \mathrm{C}_{g s}\right)$. The total cross sections as a function of the bombarding energy are shown in figure 3 . The solid curve is obtained with the use of free nucleon-nucleon cross sections. The dashed curve includes the geometrical effects of Pauli blocking. The dashed-dotted curve is the result using the Brueckner theory, and the dotted curve is the phenomenological parametrization of the free cross section.

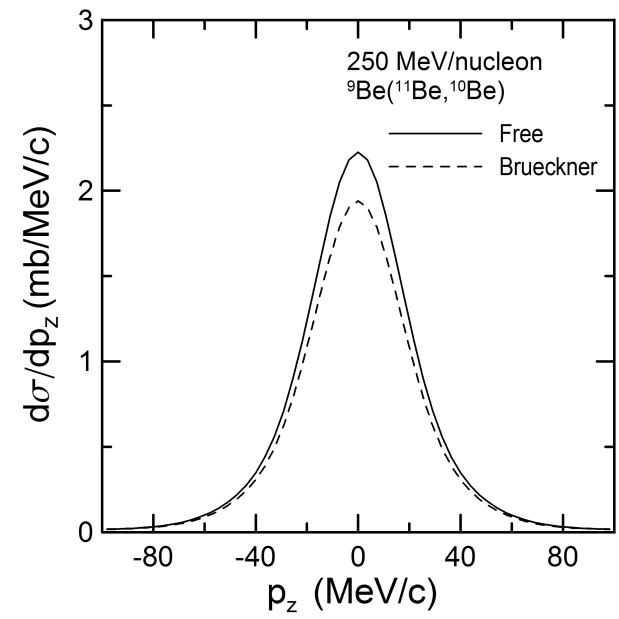

Figure 4: Longitudinal momentum distribution for the residue in the ${ }^{9} \mathrm{Be}\left({ }^{11} \mathrm{Be},{ }^{10} \mathrm{Be}\right)$, reaction at $250 \mathrm{MeV} /$ nucleon. The dashed curve is the cross section calculated using the $\mathrm{NN}$ cross section from the Brueckner theory and the solid curve is obtained the free cross section [48]. 


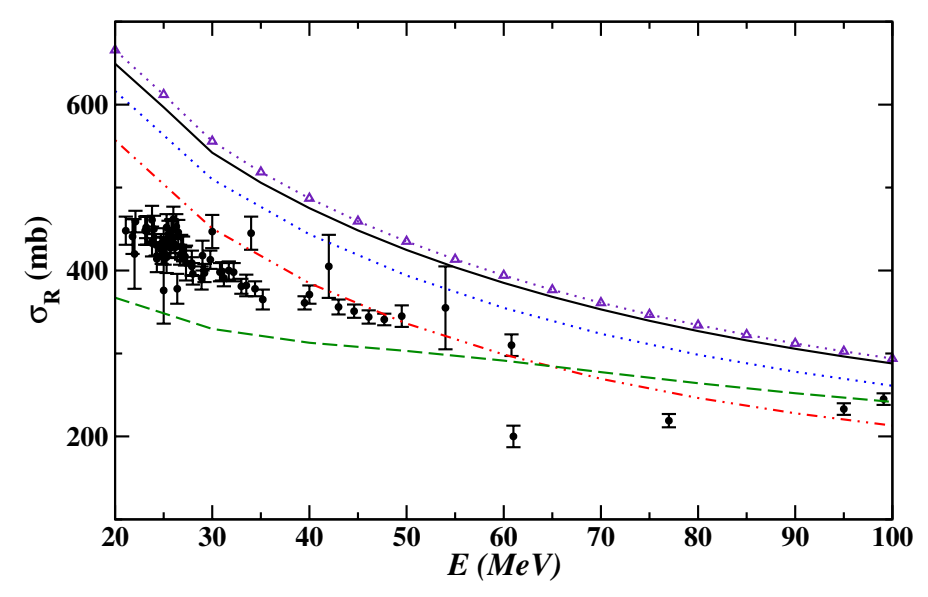

Figure 5: The total reaction cross section of the $\mathrm{p}+{ }^{12} \mathrm{C}$ taken from Ref. [52]. The curves are calculated with the free NN cross sections (solid), with a geometrical account of Pauli blocking (dashed), a phenomenological fit from Ref. [53] (dotted), and a correction from Brückner theory (dashed-dotted). The triangle-dotted curve is calculated with free $\mathrm{NN}$ cross sections but with an another HFB calculation [54] for the ${ }^{12} \mathrm{C}$ ground state density.

In figure 4 we plot the longitudinal momentum distributions for the reaction ${ }^{9} \mathrm{Be}\left({ }^{11} \mathrm{Be},{ }^{10} \mathrm{Be}\right)$, at $250 \mathrm{MeV} /$ nucleon [48]. The dashed curve is the cross section calculated using the NN cross section from the Brueckner theory and the solid curve is obtained the free cross section. One sees that the momentum distributions are reduced by $10 \%$, about the same as the total cross sections, but the shape remains basically unaltered. If one rescales the dashed curve to match the solid one, the differences in the width are not visible [49].

As we mentioned above, medium effects have been routinely neglected in the experimental analysis of knockout reactions. But their relevance has been known for a long time in the analysis of elastic and inelastic scattering, as well as of total reaction cross sections [50, 51]. The effects are larger at lower bombarding energies, where Pauli blocking strongly reduces the nucleon-nucleon cross sections in the medium. A systematic study of these effects has been presented in Ref. [51].

To corroborate these statements, in figure 5 we show the data on $\mathrm{p}+{ }^{12} \mathrm{C}$ reaction cross sections taken from the Ref. [52] in the energy region of our interest, 20-100 MeV/nucleon. The cross sections were calculated from the relation

$$
\sigma_{R}=2 \pi \int d b b\left[1-|S(b)|^{2}\right]
$$

where $S(b)$ has been calculated using an eikonal model [49] and the carbon matter density from a Hartree-Fock-Bogoliubov calculation [54]. Several distinct calculations are shown. The solid curve uses the free nucleon-nucleon cross sections and the carbon matter density from a HFB calculation [55], whereas the triangle-dotted curve (the triangles are not data, but used for better visibility) uses a different HFB density [54], consistent with the calculations presented in Ref. [56]. As expected, the agreement between the two calculations is very good.

The other curves in figure 5 show the same calculation procedure, but including medium corrections for the nucleon-nucleon cross section. The results are evidently very different than the previous ones. The dotted (dashed-dotted) [dashed] curves use phenomenological (Brueckner) [Pauli geometrical] recipes for medium effects on the cross sections. Based on the large error bars and spread of the experimental data, it is hard to judge what model adopted for medium corrections yields the best agreement with the data. It is clear that the inclusion of medium effects change the results to yield a closer reproduction of the data. 


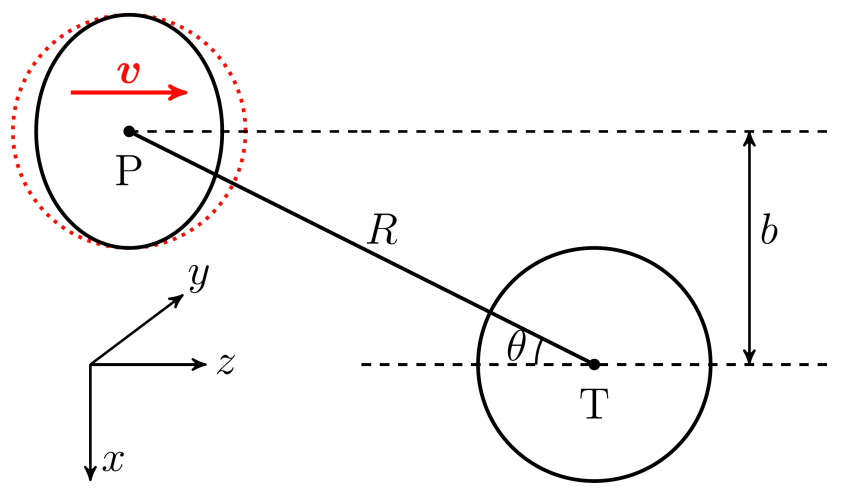

Figure 6: Two-nucleus system containing rest target and boosted projectile with the velocity v. The ellipse represents the boosted projectile density as seen from the target reference frame. $R$ and $b$ denote the distance and impact parameter between two nuclei in the target reference frame.

\section{The nucleus-nucleus interaction between boosted nuclei}

A consistent theoretical treatment of relativistic many-body systems is still a challenge in many areas of physics. The effects of the Dirac sea imply corrections due to vacuum fluctuations and other features of quantum field theory may also become manifest. An important aspect of special relativity, namely causality, is only verified if sea effects are properly incorporated. Unfortunately, up to now it is proved to be very difficult to find unambiguous experimental evidence for such effects. That might not be the case for static properties of nuclear matter for which a successful project carried out during many years has shown that nuclear energies for the ground and lower excited states of nuclei are well described by a relativistic mean-field treatment of the collection of nucleons [58, 59, 60, 61, 62, 63].

Almost nothing has been done to understand the rather relevant problem of the influence of relativity on many-body scattering. Usually, this is accounted quite straightforwardly in quantum field theory for two-body scattering including the sea effects with help of perturbation theory. But the scattering of relativistic composite objects where the compositeness becomes an active part of the scattering process is still a very difficult theoretical problem. This is an obviously important problem in nuclear physics, due to an inherent theoretical difficulty in defining a nuclear potential between many-body relativistic systems. Nonetheless, nuclear potentials are often used in the analysis of nucleus-nucleus scattering at energies of $100 \mathrm{MeV} /$ nucleon and higher. In fact, many rare isotope facilities use reactions at these energies in which relativistic effects are expected to be of the order of $10 \%$ or more for some processes of interest [2, 64, 65]. For proton-nucleus scattering a successful approach, known as "Dirac phenomenology", has been use for many years [66]. But for nucleus-nucleus collisions a reasonable account of these features has not yet been accomplished. Due to retardation, an attempt to use a microscopic description for nucleus-nucleus potentials starting from binary collisions of the constituents is not possible as a nucleus-nucleus potential requires a simultaneous interaction between all constituents. In the case of nucleus-nucleus collisions a similar approach as in the Dirac-phenomenology case can be followed up by using a mean field theory.

The nucleus-nucleus potential used in the analysis of elastic scattering experiments is a very complex object which contains information about all possible inelastic processes. The presence of inelastic channels lead to, among other things, the introduction of an imaginary part for the 
nucleus-nucleus potential. This potential is usually termed "optical potential". Although several microscopic models have been developed to calculate the optical potential, due to its complexity, it is often parametrized by a set of phenomenological functions depending on the distance between the center of mass of the interacting nuclei. The parameters set (often with ambiguities) is obtained by fitting elastic scattering data whenever available. Needless to say that for many systems (e.g. most reactions with radioactive beams) the elastic scattering data are unavailable and one has to resort to theoretical constructions of the optical potential. A popular method is to build a folding potential in which the real part of the optical potential is obtained from an integral over the nucleon-nucleon densities weighted by their individual (sometimes density dependent) interactions. The nuclear densities are taken as frozen, with all nucleons interacting simultaneously. A method to deduce the magnitude of the effects of relativity in nucleus-nucleus interactions presented in Refs. [2, 64, 65] where the nucleus-nucleus potential for a relativistic projectile was modified in a similar way as the scalar part of the electromagnetic interaction. This procedure has shown that relativistic corrections lead to appreciable changes of inelastic processes involving 100 $\mathrm{MeV} /$ nucleon projectiles. A microscopic theory to describe the covariant properties of the nuclear potential was later developed [57]. We briefly describe its main results.

\subsection{Energy density functional for two colliding nuclei}

Based on the meson exchange theory, finite nuclear systems can be well described by an energy density functional within the relativistic mean field approach $[58,59]$. In the mean field approach, the nucleons are treated as point-like particles interacting by the exchange of mesons and photons. The energy functional $[59,60,61,62,63]$ for a single nucleus, associated with $\sigma-, \omega$-, $\rho$-mesons and photon $(A)$ exchange, is given by $(\hbar=c=1)$

$E=\int d \mathbf{r} \sum_{a} \bar{\psi}_{a}(-i \boldsymbol{\gamma} \cdot \boldsymbol{\nabla}+M) \psi_{a}+\frac{1}{2} \sum_{\phi=\sigma, \omega, \rho, A} \int d \mathbf{r} d \mathbf{r}^{\prime} \sum_{a b} \bar{\psi}_{a}(\mathbf{r}) \bar{\psi}_{b}\left(\mathbf{r}^{\prime}\right) \Gamma_{\phi}\left(\mathbf{r}, \mathbf{r}^{\prime}\right) D_{\phi}\left(\mathbf{r}-\mathbf{r}^{\prime}\right) \psi_{b}\left(\mathbf{r}^{\prime}\right) \psi_{a}(\mathbf{r})$,

where the two-body interacting matrices read as

$$
\begin{array}{lc}
\Gamma_{\sigma}\left(\mathbf{r}, \mathbf{r}^{\prime}\right)= & -g_{\sigma}(\mathbf{r}) g_{\sigma}\left(\mathbf{r}^{\prime}\right), \\
\Gamma_{\omega}\left(\mathbf{r}, \mathbf{r}^{\prime}\right)= & +\left(g_{\omega} \gamma^{\mu}\right)_{\mathbf{r}}\left(g_{\omega} \gamma_{\mu}\right)_{\mathbf{r}^{\prime}}, \\
\Gamma_{\rho}\left(\mathbf{r}, \mathbf{r}^{\prime}\right)= & +\left(g_{\rho} \gamma^{\mu} \boldsymbol{\tau}\right)_{\mathbf{r}} \cdot\left(g_{\rho} \gamma_{\mu} \boldsymbol{\tau}\right)_{\mathbf{r}^{\prime}}, \\
\Gamma_{A}\left(\mathbf{r}, \mathbf{r}^{\prime}\right)=+\frac{e^{2}}{4}\left[\gamma^{\mu}\left(1-\tau_{z}\right)\right]_{\mathbf{r}}\left[\gamma_{\mu}\left(1-\tau_{z}\right)\right]_{\mathbf{r}^{\prime}} .
\end{array}
$$

In the energy functional (3.1), $\psi_{a}$ denotes stationary single particle states, $M$ is the rest mass of nucleon, and $g_{\sigma}, g_{\omega}$, and $g_{\rho}$ are the coupling constants with baryonic density dependence. The propagators of mesons ( $\phi=\sigma, \omega$, and $\rho$ and $m_{\phi}$ for the meson mass) and photon (A) are given by

$$
D_{\phi}=\frac{1}{4 \pi} \frac{e^{m_{\phi}}\left|\mathbf{r}-\mathbf{r}^{\prime}\right|}{\left|\mathbf{r}-\mathbf{r}^{\prime}\right|}, \quad D_{A}=\frac{1}{\left|\mathbf{r}-\mathbf{r}^{\prime}\right|} .
$$

Based on the energy functional (3.1), the single particle configurations $\left(\psi_{a}\right)$ can be obtained from a self-consistent iterative procedure, aiming at describing the physical properties for the ground state of the nucleus such as binding energy, radii, density distributions, etc. [59, 60, 61, 62, 63]. 
The relativistic energy functional (3.1) described above for a single nucleus can be also extended to describe the interaction between two nuclei, i.e., the interaction between a target and a boosted projectile. Here we only consider same target and projectile nuclei. As shown in figure 6, the two-nucleus system consists of a target and a projectile with velocity $\mathbf{v}$, separated by a distance $R$. For simplicity, we assume straight-line dynamics, appropriated for high-energy collisions. A given point in space is denoted by $\mathbf{r}_{t}=\left(x_{t}, y_{t}, z_{t}\right)$ in the frame of reference of the target with respect to its center of mass. For a collision with impact parameter $b$ this point can be related to its coordinate $\mathbf{r}_{p}=\left(x_{p}, y_{p}, z_{p}\right)$ in the frame of reference of the projectile by means of a Lienard-Wiechert transformation. If $\mathbf{v}$ is taken as the $z$ axis, this transformation reads

$$
\begin{aligned}
& x_{p}=x_{t}+b, \quad x_{t}=x_{p}-b, \\
& y_{p}=y_{t}, \quad y_{t}=y_{p}, \\
& z_{p}=\gamma\left(z_{t}+R \cos \theta\right), \quad z_{t}=\gamma\left(z_{p}-R \cos \theta\right),
\end{aligned}
$$

where the impact parameter is related to the relative distance between the center of mass of the nuclei by $b=R \sin \theta$. The Lorentz factor is $\gamma=\left(1-\beta^{2}\right)^{-1 / 2}$ and $\beta=v / c$.

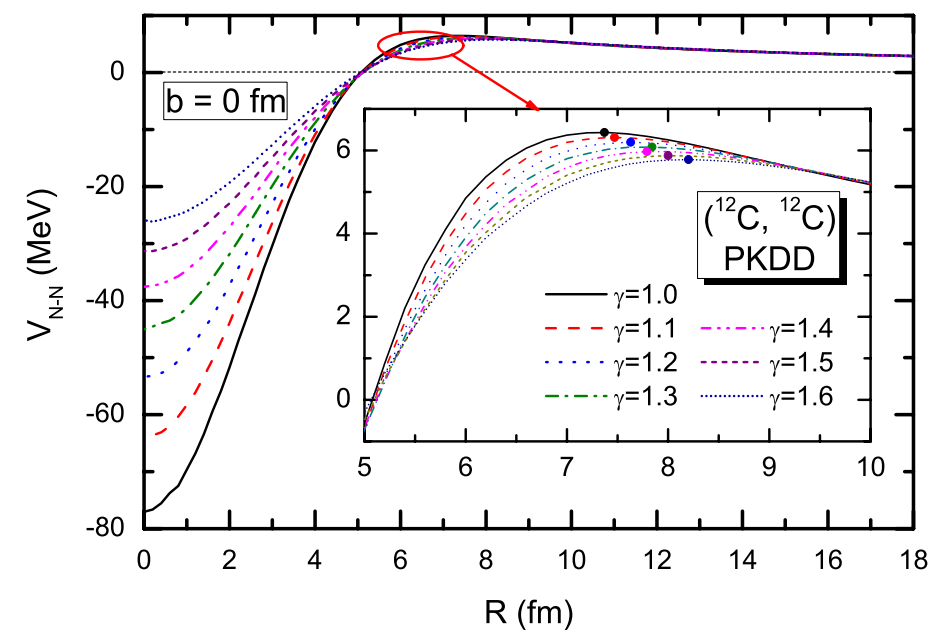

Figure 7: The interaction potentials $(\mathrm{MeV})$ as functions of the distance $R$ (fm) between rest target and boosted projectile nuclei for impact parameter $b=0 \mathrm{fm}$ and different values of $\gamma$. The inset presents the potentials from $R=5$ $\mathrm{fm}$ to $10 \mathrm{fm}$ with an enlarged scale.

The total energy functional for two-nucleus system shown in figure 6 is given by

$$
E\left(A_{t}, A_{p}, \mathbf{v}\right)=E\left(A_{t}\right)+E\left(A_{p}, \mathbf{v}\right)+\mathbb{E}\left(A_{t}, A_{p}, \mathbf{v}\right),
$$

where $E\left(A_{t}\right)$ and $E\left(A_{p}, \mathbf{v}\right)$ denote respectively the target (with mass number $\left.A_{t}\right)$ and the projectile (with mass number $\left.A_{p}\right)$ energy functional, and $\mathbb{E}\left(A_{t}, A_{p}, \mathbf{v}\right)$ represents the interacting potential energy. For the isolated target and projectile, the energy functional $E\left(A_{t}\right)$ and $E\left(A_{p}, \mathbf{v}\right)=E\left(A_{p}\right)$ can be determined from Eq. (3.1). Similar as the second term in right hand of Eq. (3.1), the interacting one $\mathbb{E}\left(A_{t}, A_{p}, \mathbf{v}\right)$ can be obtained as

$$
\mathbb{E}\left(A_{t}, A_{p}, \mathbf{v}\right)=\sum_{\phi=\sigma, \omega, \rho, A} \int d \mathbf{r} \int d \mathbf{r}^{\prime} \sum_{a b} \bar{\psi}_{t, a}(\mathbf{r}) \bar{\psi}_{p, b}\left(\mathbf{r}^{\prime}\right) \Gamma_{\phi}\left(\mathbf{r}, \mathbf{r}^{\prime}\right) D_{\phi}\left(\mathbf{r}-\mathbf{r}^{\prime}\right) \psi_{p, b}\left(\mathbf{r}^{\prime}\right) \psi_{t, a}(\mathbf{r}),
$$

where the integral over $\mathbf{r}$ and $\mathbf{r}^{\prime}$ are done within the same reference frame, e.g., the target inertial frame. The single particle configurations $\psi_{t, a}$ and $\psi_{p, b}$ (subindex $t$ for target and $p$ for projectile) can 
be determined from the individual self-consistent calculations in the target and projectile frames, respectively. Because the collisions are fast, we adopt a frozen configuration scheme in which the rearrangement effects from the nucleus-nucleus interaction are neglected in determining the single configurations of each nucleus.

Taking $\sigma$ and $\omega$ as example, the corresponding contributions in Eq. (3.9) can be written as,

$$
\begin{aligned}
& \mathbb{E}_{\sigma}=-\frac{1}{\gamma} \int d \mathbf{r}_{t} \int d \mathbf{r}_{p}^{\prime} g_{\sigma}\left(\mathbf{r}_{t}\right) \rho_{s, t}\left(\mathbf{r}_{t}\right) D_{\sigma}\left(\mathbf{r}_{t}-\mathbf{r}_{t}^{\prime}\right) \rho_{s, p}\left(\mathbf{r}_{p}^{\prime}\right) g_{\sigma}\left(\mathbf{r}_{p}^{\prime}\right), \\
& \mathbb{E}_{\omega}=+\int d \mathbf{r}_{t} \int d \mathbf{r}_{p}^{\prime} g_{\omega}\left(\mathbf{r}_{t}\right) \rho_{b, t}\left(\mathbf{r}_{t}\right) D_{\omega}\left(\mathbf{r}_{t}-\mathbf{r}_{t}^{\prime}\right) \rho_{b, p}\left(\mathbf{r}_{p}^{\prime}\right) g_{\omega}\left(\mathbf{r}_{p}^{\prime}\right) .
\end{aligned}
$$

The scalar and baryonic densities $\rho_{s}$ and $\rho_{b}$ can be obtained as

$$
\rho_{s}(\mathbf{r})=\sum_{a} \bar{\psi}_{a}(\mathbf{r}) \psi_{a}(\mathbf{r}), \rho_{b}(\mathbf{r})=\sum_{a} \bar{\psi}_{a}(\mathbf{r}) \gamma^{0} \psi_{a}(\mathbf{r}),
$$

where the sums are restricted within one nucleus.

In the interacting energy functional (3.9), there exist two types of couplings, the scalar $(\sigma)$ and the vectors $(\omega, \rho$, and $A)$. For a straight-line motion, the Lorentz boots are: $\left\{\rho_{b, p}\left(\mathbf{r}_{p}\right), 0\right\} \rightarrow$ $\left\{\gamma \rho_{b, p}\left(\mathbf{r}_{p}\right), \gamma \rho_{b, p}\left(\mathbf{r}_{p}\right) \beta\right\}$. The scalar coupling remains invariant under the Lorentz transformations because $d \mathbf{r}_{t}^{\prime}=d \mathbf{r}_{p}^{\prime} / \gamma$ and the Lorentz contraction of the longitudinal distance cancels out the Lorentz enhancement of the field. This contrasts with the vector coupling channels where the Lorentz transformation leads to a strong dependence on the projectile velocity.

In energy functional (3.9), the coupling constants are functions of density $\rho_{v}=\sqrt{j^{\mu} j_{\mu}}$ and $j^{\mu}=\bar{\psi}_{t} \gamma \psi_{t}^{\mu}+\bar{\psi}_{p} \gamma^{\mu} \psi_{p}$, and the Lorentz transformation from the target to projectile, and the reverse, leads to

$$
\begin{array}{r}
\rho_{v}\left(\mathbf{r}_{t}\right)=\sqrt{\rho_{b, t}^{2}\left(\mathbf{r}_{t}\right)+2 \gamma \rho_{b, t}\left(\mathbf{r}_{t}\right) \rho_{b, p}\left(\mathbf{r}_{p}\right)+\rho_{b, p}^{2}\left(\mathbf{r}_{p}\right)}, \\
\rho_{v}\left(\mathbf{r}_{p}\right)=\sqrt{\rho_{b, t}^{2}\left(\mathbf{r}_{t}\right)+2 \gamma \rho_{b, t}\left(\mathbf{r}_{t}\right) \rho_{b, p}\left(\mathbf{r}_{p}\right)+\rho_{b, p}^{2}\left(\mathbf{r}_{p}\right)} .
\end{array}
$$

The method described above allows us to calculate the interaction potential of the two nuclei in terms of the single-particle states calculated within a relativistic mean field theory. In practice, the calculations are very complicated because the advantage of treating wavefunctions and densities in terms of angular momentum expansions in spherical basis is lost. Due to Eq. 3.7, the boosted quantities also include Lorentz $\gamma$ factors within the polar angles of the single-particle states for the projectile rendering a very complicated description of their radial and angular dependence.

\subsection{The boosted nucleus-nucleus potential}

We take ${ }^{12} \mathrm{C}-{ }^{12} \mathrm{C}$ as a typical example of a two-nucleus system in figure 6 and study the nucleus-nucleus interacting potential energy according to the method described above. In the first step, each nucleus is treated in their reference frame independently to obtain the single particle configurations, from which the nucleus-nucleus interacting potential energy is determined via Eq. (3.9). The effective interaction PKDD [68] is utilized to determine the single particle configurations as well as the nucleus-nucleus potential.

In figure 7 we show the nucleus-nucleus potential energies $V(R)=\mathbb{E}$ [see Eq. (3.9)] as a function of the distance $R$ for different values of Lorentz factor $\gamma$. The impact parameter is set as $b=$ 


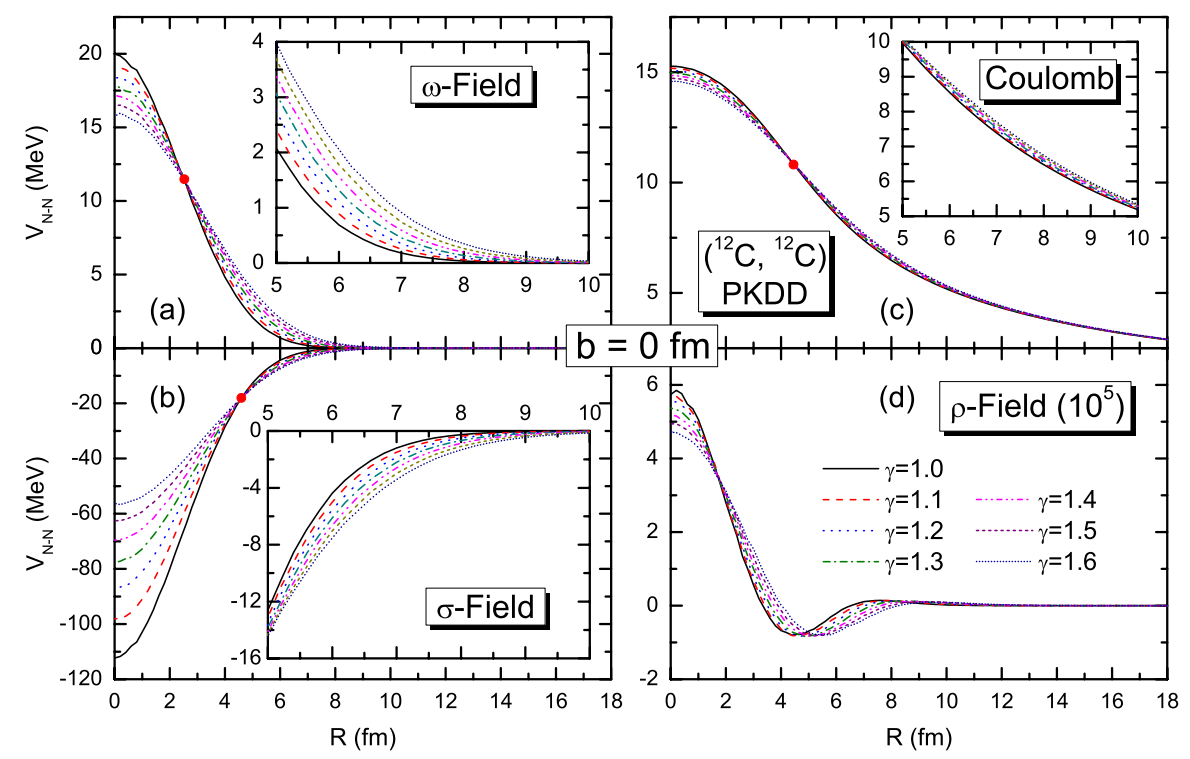

Figure 8: Contributions to the interaction potentials from the $\omega$ (a), $\sigma$ (b), Coulomb (c), and $\rho$ (d) fields are shown as functions of distance $R(\mathrm{fm})$ with different values of $\gamma$ for the same two-nucleus system shown in the previous figure. The insets show the details from $R=5 \mathrm{fm}$ to $10 \mathrm{fm}$ with enlarged scales.

$0 \mathrm{fm}$, and only the surface part of the potential has any useful application. The inset gives the results from $R=5$ to $10 \mathrm{fm}$ with enlarged scale. The nucleus-nucleus potential gets contributions from different fields at different times. First the nuclei approach from a fairly large distance, there exist very little overlap between the target and projectile densities and the potential is mainly contributed by the long range interaction - the photon field $(A)$, which yields the Coulomb barrier. As the distance decreases to, e.g., $R \sim 7.5 \mathrm{fm}$ for $\gamma=1.0$, the densities start to overlap and the nucleusnucleus potential bend toward a negative value. This is due the contribution of the meson fields leading to attraction. When $R \leq 5 \mathrm{fm}$, the nucleus-nucleus potential have only net attractions. For the chosen nucleus ${ }^{12} \mathrm{C}$, the self-consistent calculation with PKDD gives the neutron, proton and total radii, respectively, as $r_{n}=2.26 \mathrm{fm}, r_{p}=2.28 \mathrm{fm}$, and $r=2.27 \mathrm{fm}$. These values (multiplied by 2 ) are consistent with the evolution of the nucleus-nucleus potential with respect to separation distance $R$, as seen in figure 7 .

The nucleus-nucleus potentials shown in figure 7 also display a systematical behavior with respect to the Lorentz factor $\gamma$ which represents the boosted energy of the projectile, roughly ( $\gamma-$ 1) $M$ per nucleon. With increasing bombarding energy, the nucleus-nucleus potential well becomes shallower, as seen in figure 7. The point where the potential becomes attractive does not change appreciably with the bombarding energy. But, as shown in the inset, the potential barrier does have a strong dependence on the bombarding energy. This hints to a destructive additional (because of the boost) cancellation between the attractive meson fields and the repulsive photon field. These additional cancellations also tend to lead to a shallower potential. The filled circles in the inset show the tendency of this effect to stretch the nucleus-nucleus potential outwards as the energy increases.

In order to have better understanding about the systematics of the nucleus-nucleus potentials, 
we show in figure 8 the detailed contributions from four coupling channels, (a) $\omega$-vector, (b) $\sigma$ scalar, (c) Coulomb vector, and (d) $\rho$-vector couplings. The corresponding insets present the results from $R=5$ to $10 \mathrm{fm}$ with enlarged scales. In figure 8 (d), it is clearly seen that the contribution from the $\rho$ field is very tiny since in ${ }^{12} \mathrm{C}$ the nucleons are isospin saturated. Among the contributions to the nucleus-nucleus potential, the attraction mainly originates from the $\sigma$ scalar coupling, whereas the repulsion is mainly due to the $\omega$ and Coulomb fields. Starting from a fairly large separation distance, the sole contribution is the photon field which yields an increasing Coulomb repulsion. At $R \sim 10 \mathrm{fm}$ the $\sigma$ and $\omega$ fields start to act with distinct contributions to turn the Coulomb repulsion into nuclear attraction. From figure 8(a) and (b), one sees that the $\omega$ and $\sigma$ contributions increase rapidly when the target and projectile approach. This is mainly due to the short range character of the meson exchange interaction.

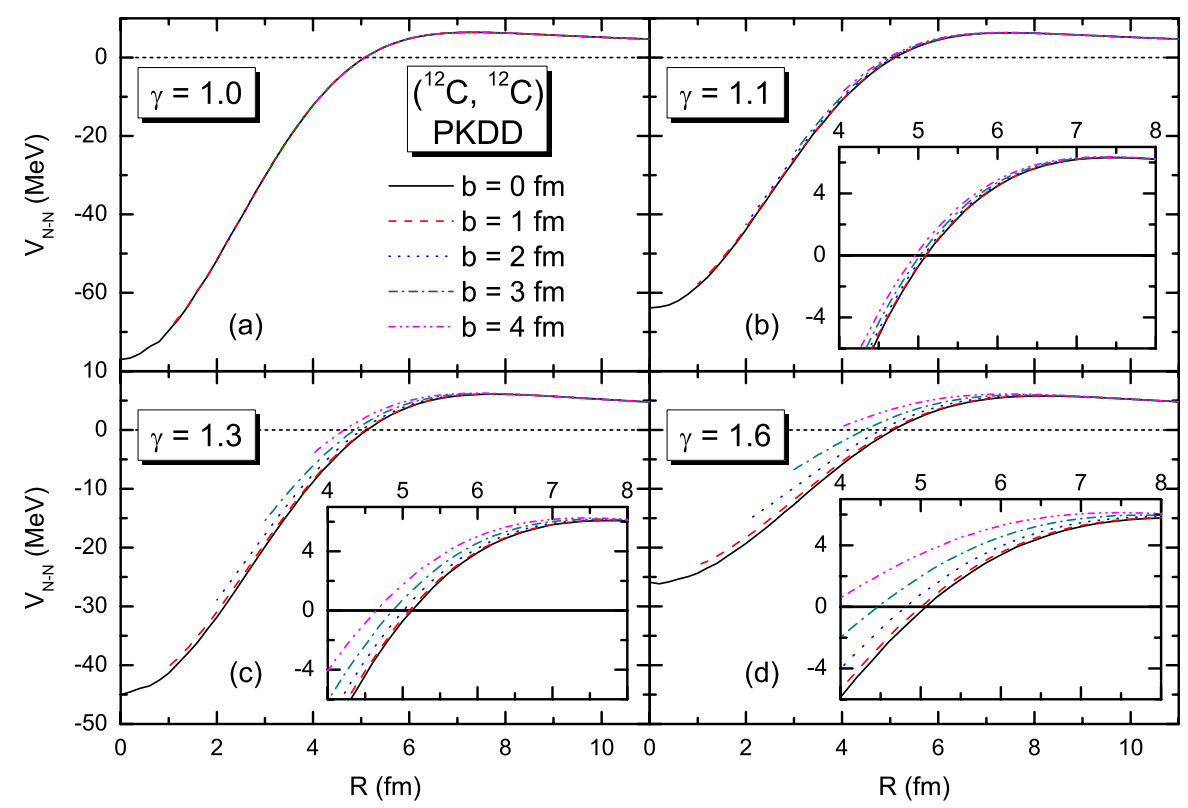

Figure 9: The nucleus-nucleus interaction potentials (in $\mathrm{MeV}$ ) are shown as function of the distance $R$ between the target and projectile nuclei for different values of $\gamma[$ (a) $\gamma=1.0$; (b) $\gamma=1.1$; (c) $\gamma=1.3$; and (d) $\gamma=1.6]$ and different impact paramters $b(\mathrm{fm})$. The insets show the results from $R=3.5 \mathrm{fm}$ to $8 \mathrm{fm}$ with enlarged scales.

As seen from the inset of figure 8 (c), the photon field has a rather weak dependence on the boosted energy whereas a stronger dependence is found from the contributions from $\sigma$ and $\omega$ fields. As shown in the insets of figure 8 (a) and (b), the repulsive ( $\omega$ field) and attractive ( $\sigma$ field) increase with the boosted energy considerably. When the target and projectile approach a close distance, the curves with different bombarding energies tend to cross each another at some point, denoted by red circles in figure 8 . In fact these are turning points, where the trend of the bombarding energy dependence is just reversed. Similar turning points can be also found in figure 7 , where the nucleus-nucleus potential becomes attractive.

In figures 7 and 8 , the impact parameter $b$ is set to zero. In figure 9 we show the nucleusnucleus potential as a function of the separation distance $R$ with the impact parameter $b=0,1,2,3$, 
and $4 \mathrm{fm}$. For comparison, the results with four bombarding energies are shown in figure 9, (a) $\gamma=0$, (b) $\gamma=1.1$, (c) $\gamma=1.3$, and (d) $\gamma=1.6$. As seen from figure 9 (a) and (b), the nucleus-nucleus potentials with lower bombarding energy are not sensitive to the impact parameter. With increasing bombarding energy, the potentials show a strong dependence on the impact parameter. As shown in figure 9 (c) and (d), the slope of the nucleus-nucleus potential at large distances becomes smaller with increasing impact parameter. It is also demonstrated by the insets in figure 9.

In order to asses the relativistic modifications of the nucleus-nucleus potential on scattering observables, we consider the elastic scattering cross section of spherically symmetric nuclei. Our results for ${ }^{12} \mathrm{C}+{ }^{12} \mathrm{C}$ scattering are compared to experimental data at bombarding energies of 85 $\mathrm{MeV} /$ nucleon $200 \mathrm{MeV} /$ nucleon in figure 10 (solid lines) [69]. We compare the cross sections with those calculated with non-relativistic dynamics are shown in figure 10 by dashed lines. WE assume an imaginary part of the optical potential having the same form as the real part, with a scaling parameter $\lambda$, which is set to 0.5 (0.4) for the bombarding energy of 85 (200) Mev per nucleon. One observes that the minima of the elastic cross sections are shifted to smaller values of the scattering angle. This is due to the smaller effective radius of the nucleus-nucleus potential when relativistic effects are included, as observed in figures 7-9. For a discussion of the effects of size and diffuseness of optical potentials on scattering observables, see, e.g., Ref. [70]. The diffuseness of the potentials also increases with the inclusion of relativistic effects. This is shown manifest in figure 10 by a (slightly) faster decrease of the cross section as a function of angle. We also notice that the relativistic effects become more pronounced at larger bombarding energies, as expected.

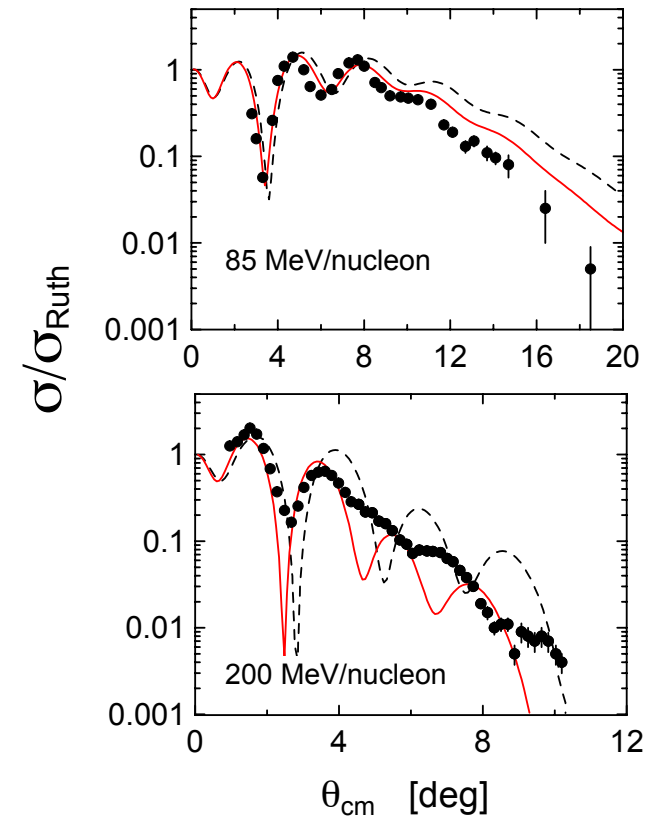

Figure 10: Elastic cross sections $d \sigma / d \Omega=|f(\vartheta)|^{2}$ in units of Rutherford cross sections $\left|f_{\text {Ruth. }}(\vartheta)\right|^{2}$ as functions of the scattering angle $\vartheta$, with different values of Lorentz factor $\gamma$. The dashed lines represent the results with non-relativistic reduction.

\section{Conclusions}

There were many questions not addressed in this review, such as the role of central nucleusnucleus collisions in determining phase transition, equation of state, and a quark-gluon plasma, 
all topics or relevance in astrophysics. The review was more focused on the role of short-lived, exotic nuclei. There are many important scientific questions to be addressed both experimentally and theoretically in nuclear physics of exotic nuclei with relevance for astrophysics. These questions provide extreme challenges for experiments and theory. On the experimental side, producing the beams of radioactive nuclei needed to address the scientific questions has been an enormous challenge. Pioneering experiments have established the techniques and present-generation facilities have produced first exciting science results, but the field is still at the beginning of an era of discovery and exploration that will be fully underway once the range of next- generation facilities becomes operational. The theoretical challenges relate to wide variations in nuclear composition and rearrangements of the bound and continuum structure, sometimes involving near-degeneracy of the bound and continuum states. The extraction of reliable information from experiments requires a solid understanding of the reaction process, in addition to the structure of the nucleus. In astrophysics, new observations, for example the expected onset of data on stellar abundances, will require rare-isotope science for their interpretation.

One of the big challenges is the investigation of relativistic effects in nucleus-nucleus potentials. We have shown here an example, based on the relativistic mean field theory [57]. The relativistic effects have been studied by analyzing the dependence of the potentials upon bombarding energies and impact parameters. It is found that for a given impact parameter the Coulomb barrier is softened with increasing bombarding energy. For large bombarding energies, the potential edge becomes increasingly flat with increasing impact parameters which indicates that the target and projectile have to get closer to compensate for more attraction. We used the relativistic corrected potentials in the analysis of elastic scattering of ${ }^{12} \mathrm{C}+{ }^{12} \mathrm{C}$ at bombarding energies of 85 $\mathrm{MeV} /$ nucleon $200 \mathrm{MeV} /$ nucleon. The imaginary part of the optical potential was assumed to have the same form as the real part, with a scaling factor. Although not perfect, the agreement is rather reasonable in view of the simplifications adopted to obtain the optical potential.

The studies carried out here are exploratory in the sense that a consistent theory of a nucleusnucleus potential including relativity requires a much more elaborated effort. Such a theory is in fact missing in the literature. To be consistent, this theory would have to include other effects such as exchange terms, which are missing in our calculation, or the effects of multiple scattering which should also become relevant at increasing energies. Both mentioned effects are challenging: (a) the first involves the calculation of complex multidimensional integrals and (b) the second involves handling retardation effects in multiple, sequential, collisions. Many of nucleus-nucleus experiments at energies of $100 \mathrm{MeV} /$ nucleon and above are presently been carried out around the world with the goal to extract spectroscopic information on rare nuclear species [70]. Our work shows that some modifications on the values of the extracted spectroscopic quantities might occur due to the relativistic dynamics missing in most methods used to construct a nucleus-nucleus potential, such as the folding models. An accurate assessment of such modifications is worthwhile to pursue.

\section{References}

[1] L.F. Canto, P.R.S. Gomes, R. Donangelo, M.S. Hussein, Phys. Rep. 424, 1 (2006).

[2] C.A.Bertulani, Phys. Rev. Lett. 94, 072701 (2005). 
[3] C.A. Bertulani, Phys. Rev. C49 (1994) 2688; Nucl. Phys. A587 (1995) 318; Z. Phys. A356 (1996) 293.

[4] A.M. Mukhamedzhanov and N.K. Timofeyuk, JETP Lett. 51, 282 (1990).

[5] Y.C. Tang, M. LeMere and D.R. Thompsom, Phys. Rep. 47, 167 (1978).

[6] Sofia Quaglioni and Petr Navrátil, Phys. Rev. Lett. 101, 092501 (2008).

[7] C.A. Bertulani, Rev. Mex. Phys. 53, 11 (2008).

[8] P. Navrátil, C.A. Bertulani, and E. Caurier, Phys. Lett. B 634 (2006) 191; Phys. Rev. C 73 (2006) 065801.

[9] Petr Navratil, Robert Roth, Sofia Quaglioni, Ab initio many-body calculation of the 7Be(p,gamma)8B radiative capture, arXiv: 1105.5977.

[10] B. K. Jennings, S. Karataglidis, and T. D. Shoppa, Phys. Rev. C 58, 579 (1998).

[11] R. D. Williams and S. E. Koonin, Phys. Rev. C 23, 2773 (1981).

[12] E.G. Adelberger et al., Rev. Mod. Phys. 83, 195 (2011).

[13] G. Baur, Phys. Let. B178 (1986) 135.

[14] M. La Cognata et al., Phys. Rev. C 76, 065804 (2007).

[15] R.G. Pizzone et al, Few-Body Systems 50, 319 (2011).

[16] A. M. Mukhamedzhanov (unpublished).

[17] S. Cherubini et al., Astrophys. J. 457, 855 (1996).

[18] M. Zadro, D. Miljanińc, C. Spitaleri, G. Calvi, M. Lattuada, and F. Riggi, Phys. Rev. C 40, 181 (1989).

[19] C. Spitaleri et al., Phys. Rev. C 63, 055801 (2001).

[20] M. Lattuada et al., Astrophys. J. 562, 1076 (2001).

[21] G. Pizzone,et al., Phys. Rev. C 87, 025805 (2013).

[22] A. Tumino et al., Phys. Lett. B 705, 546 (2011).

[23] R.E. Tribble et al., Proceedings of Science (online at: http://pos.sissa.it/index.html), 2007.

[24] A.M. Mukhamedzhanov et al, J. Phys. Conference Series 202, 012017 (2010).

[25] C.E.Aguiar, A.N.F.Aleixo and C.A.Bertulani, Phys. Rev. C 42, 2180 (1990).

[26] C.A. Bertulani and A.M. Nathan, Nucl. Phys. A 554 (1993) 158.

[27] C.A. Bertulani, A. Stuchbery, T. Mertzimekis and A. Davies, Phys. Rev. C 68, 044609 (2003).

[28] K. Alder and A. Winther, Electromagnetic Excitation, North-Holland, Amsterdam, 1975.

[29] A. Winther and K. Alder, Nucl. Phys. A 319 (1979) 518.

[30] A.N.F. Aleixo and C.A. Bertulani, Nucl. Phys. A 505 (1989) 448.

[31] C.A. Bertulani, C.M. Campbell, and T. Glasmacher, Comput. Phys. Commun. 152, 317 (2003).

[32] T. Glasmacher, Nucl. Phys. A 693 (2001) 90.

[33] C.A. Bertulani, Proceedings of Workshop on "Reaction Mechanisms for Rare Isotope Beams" Michigan State University March 9-12,2005, arXiv.org:nucl-th/0505015. 
[34] C.A.Bertulani and G.Baur, Phys. Reports 163 (1988) 299.

[35] G. Baur, C. Bertulani and H. Rebel, Nucl. Phys. A459 (1986) 188.

[36] T. Motobayashi, et al., Phys. Rev. Lett. 73 (1994) 2680.

[37] H. Esbensen, G.F. Bertsch, and K. Snover, Phys. Rev. Lett. 94,042502 (2005); Moshe Gai, Phys. Rev. Lett. 96, 159201 (2006); H. Esbensen, G.F. Bertsch, and K. Snover, Phys. Rev. Lett. 94, 042502 (2005).

[38] C.A. Bertulani and M. Gai, Nucl. Phys. A636 (1998) 227.

[39] C.A. Bertulani and L.F. Canto, Nucl. Phys. A 539 (1992) 163

[40] G. Baur, C.A. Bertulani and D.M. Kalassa, Nucl. Phys. A550 (1992) 527.

[41] G.F. Bertsch and C.A. Bertulani, Nucl. Phys. A556 (1993) 136; Phys. Rev. C49 (1994) 2834 ; H. Esbensen, G.F. Bertsch and C.A. Bertulani, Nucl. Phys. A581 (1995) 107.

[42] M. Gai and C.A. Bertulani, Phys. Rev. C52 (1995) 1706; Nucl. Phys. A636 (1998) 227.

[43] H. Esbensen and G. Bertsch, Nucl. Phys. A600 (1996) 37.

[44] C.A. Bertulani, Phys. Lett. B3 (2002) 205.

[45] C. A. Bertulani and K. W. McVoy, Phys. Rev. C 46, 2638 (1992).

[46] R. Anne, et al., Nucl. Phys. A 575 (1994) 125.

[47] C. A. Bertulani and P. G. Hansen, Phys. Rev. C 70, 034609 (2004).

[48] C.A. Bertulani and C. De Conti, Phys. Rev. C 81, 064603 (2010).

[49] M. Karakoç, A. Banu, C. A. Bertulani, and L. Trache, Phys. Rev. C 87, 024607 (2013).

[50] L. Ray, Phys. Rev. C 20, 1857 (1979).

[51] M.S. Hussein, R.A. Rego and C.A. Bertulani, Phys. Rep. 201 (1991) 279.

[52] R. F. Carlson, At. Data Nucl. Data Tables 63, 93 (1996).

[53] Cai Xiangzhou, Feng Jun, Shen Wenqing, Ma Yugang, Wang Jiansong, and Ye Wei, Phys. Rev. C58, 572 (1998).

[54] M. Beiner and R.J. Lombard, Ann. Phys. N.Y. 86, 262 (1974); F. Carstoiu and R.J. Lombard, ibid. 217, 279 (1992).

[55] http://www.nscl.msu.edu/ brown/resources/resources.html

[56] L. Trache, F. Carstoiu, A. M. Mukhamedzhanov, and R. E. Tribble, Phys. Rev. C 66, 035801 (2002).

[57] Wen-Hui Long and C.A. Bertulani, Phys. Rev. C 83, 024907 (2011).

[58] J. D. Walecka, Ann. Phys. (NY) 83, 491 (1974).

[59] B. D. Serot and J. D. Walecka, Adv. Nucl. Phys. 16, 1 (1986).

[60] P.-G. Reinhard, Rep. Prog. Phys. 52, 439 (1989).

[61] P. Ring, Prog. Part. Nucl. Phys. 37, 193 (1996).

[62] M. Bender, P.-H. Heenen, and P.-G. Reinhard, Rev. Mod. Phys. 75, 121 (2003).

[63] J. Meng, H. Toki, S. G. Zhou, S. Q. Zhang, W. H. Long, and L. S. Geng, Prog. Part. Nucl. Phys. 57, 470 (2006). 
[64] K. Ogata and C.A. Bertulani, Prog. Theor. Phys. (Letter) 121, 1399 (2009).

[65] K. Ogata and C.A. Bertulani, Prog. Theor. Phys. 123, 701 (2010).

[66] L.G. Arnold and B.C. Clark, Phys. Lett. B 84, 46 (1979).

[67] R. Brockmann and H. Toki, Phys. Rev. Lett. 68, 3408 (1992).

[68] W. Long, J. Meng, N. Van Giai, and S.-G. Zhou, Phys. Rev. C 69, 034319 (2004).

[69] M. Buenerd et al., Phys. Rev. C 26, 1299 (1982).

[70] C.A. Bertulani and A. Gade, Physics Reports 485, 195 (2010). 